\title{
3D Collimation of Matter Waves
}

\author{
An innovative matter-wave lens exploiting atomic interactions is able to \\ slow the expansion of a Bose-Einstein condensate in three dimensions, \\ thus reaching unprecedented ultralow temperatures.
}

\section{By Vincenzo Tamma}

$\Lambda$ t ultralow temperatures, dilute atomic gases manifest their full quantum nature as matter waves in the form of Bose-Einstein condensates (BECs). Through the interference of matter waves in an interferometer, researchers

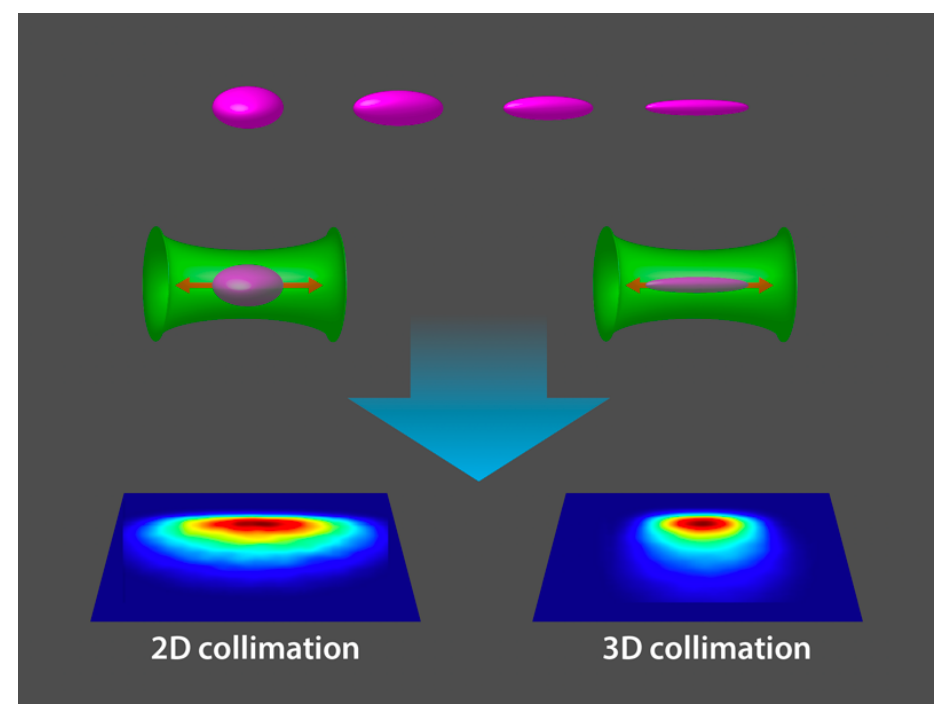

Figure 1: The new 3D collimation technique begins by inducing a collective mode oscillation in the Bose-Einstein condensate (BEC) due to atomic interactions (top). The BEC is released once it has collapsed around its axis (right), leading to a reduced expansion along that same axis (red arrows). To reduce the expansion in the other two dimensions, a cylindrical magnetic lens (qualitatively shown in green) is applied after $80 \mathrm{~ms}$, when the interaction energy has been converted into kinetic energy. Full 3D collimation is observed after free fall via absorption imaging of the BEC (bottom). For comparison, 2D collimation (no induced oscillations but only magnetic lens) is shown on the left.

Credit: APS/Alan Stonebraker can probe gravitational effects at microscopic scales and thereby test gravity at the quantum level. But improving the precision of these tests requires lowering the temperature of the BECs even further. Ernst Rasel from Leibniz University Hannover in Germany and colleagues have realized BECs at the lowest temperature so far (38 pK) by collimating the atoms in 3D with a new time-domain lens system based on atomic interactions [1].

The team prepared BEC matter waves with over one hundred thousand atoms and recorded their time evolution via absorption imaging during $2 \mathrm{~s}$ of free fall in a 110-m-high tower. Without any lensing applied, the BEC expanded through random thermal motion and became too dilute to be detected after $160 \mathrm{~ms}$. In contrast, when the team collimated the atoms with their lens, the expansion slowed, and the BEC was visible throughout its fall. Moreover, the authors extrapolated their results and found that their innovative collimation technique can generate slowly expanding BECs that should remain detectable even after $17 \mathrm{~s}$, which could be useful in future tests of gravity in space-based experiments.

BEC matter waves are a magnificent tool with which to explore the interface between quantum theory and general relativity-the underlying theories of the microcosmos and the macrocosmos, respectively. When a BEC is placed in an interferometer, its interference pattern will partly depend on gravitational effects due to the mass of the atoms. Detecting these effects could allow for fundamental tests, such as the verification of the Einstein equivalence principle with quantum objects. These tests require letting the BEC freely evolve for long times, which poses a problem, as the atoms tend to fly apart because of the internal kinetic energy (or temperature) of the system. Reducing this energy would extend the expansion 
time before the BEC becomes too dilute and improve the precision of matter-wave interferometry.

A powerful way to reduce a BEC's internal kinetic energy is to exploit a matter-wave lens to focus the BEC atoms at infinity. Standard matter-wave lenses that are based on magnetic, optical, or electrostatic forces have indeed been used to reduce the BEC internal kinetic energy. Those tools can reach effective temperatures of about 50 pK but, unfortunately, only in two dimensions [2]. A magnetic lens, for example, has a cylindrical geometry that can bend the trajectory of atoms inward along the two radial directions, but it lacks this refractive power along the axial direction.

In their experiments, Rasel and colleagues achieve an unprecedently low temperature of $38 \mathrm{pK}$ by exploiting an innovative matter-wave lens system in the time domain. Such a system can focus the BEC wave at infinity in all three spatial dimensions by cleverly combining both a magnetic lens and a collective-mode excitation (or shape vibration) in the BEC [3].

The team first generated a BEC of approximately one hundred thousand rubidium atoms within a cylindrically shaped magnetic trap produced on a microchip [4]. To excite the collective-mode oscillation, the researchers quickly reduced the trap magnetic bias field along one direction, while increasing the trapping strength in the other two directions. Because of the atomic interactions, the $B E C$ responded by lengthening along its axis and slimming around the waist (Fig. 1). If allowed to continue this oscillation, the BEC would return to its original shape, but the researchers instead released the BEC at the time of maximum slimming. This was the key step for achieving 3D collimation, as it minimized the expansion along the axial direction. To slow the expansion around the BEC's waist, the team applied a magnetic lens that collimated the atomic motion in the other two dimensions.

The experiments were performed at the Bremen drop tower in Germany, which provides an exceptional microgravity environment with residual accelerations of the order of $10^{-6} \mathrm{~g}$ [5]. The researchers released the BEC at the top of the tower and measured its size via absorption imaging at different points during the free fall. From the data, they surmised that the expansion velocities were of the order of $60 \mu \mathrm{m} / \mathrm{s}$. In simulations, the team extended the free-fall time and showed that the BEC should remain detectable for up to $17 \mathrm{~s}$.

By tuning both the oscillation time at the condensate's release and the strength of the magnetic lens's potential, this new lensing method offers the possibility to engineer and control BEC shape and expansion for fundamental physics tests as well as for quantum sensing technologies. Indeed, the ability to generate slowly expanding BECs for tens of seconds can enable high-precision gravitational-wave detection [6], measurements of the gravitational constant [7] and the tidal force of gravity [8], as well as the search for ultralight dark matter [9] and a stringent quantum verification of Einstein's equivalence principle, both in drop towers and in space [10].

Furthermore, the 3D matter-wave lens system introduced by Rasel and co-workers provides a new and exciting perspective on the quantum advantage hidden behind the presence of interatomic interactions, often viewed as a drawback in matter-wave optics with long expansion times. Indeed, such interactions can be exploited as a powerful metrological tool in the development of matter-wave quantum sensors, enabling not only high-coherence properties but also highly nonclassical correlations.

Vincenzo Tamma: School of Mathematics and Physics and Institute of Cosmology and Gravitation, University of Portsmouth, Portsmouth, United Kingdom

\section{REFERENCES}

1. C. Deppner et al., "Collective-mode enhanced matter-wave optics," Phys. Rev. Lett. 127, 100401 (2021).

2. T. Kovachy et al., "Matter wave lensing to picokelvin temperatures," Phys. Rev. Lett. 114, 143004 (2015).

3. D. S. Jin et al., "Collective excitations of a Bose-Einstein condensate in a dilute gas," Phys. Rev. Lett. 77, 420 (1996); M.-O. Mewes et al., "Collective excitations of a Bose-Einstein condensate in a magnetic trap," Phys. Rev. Lett. 77, 988 (1996).

4. Y. T. Baiborodov et al., "An adiabatic trap with combined magnetic field,” J. Nucl. Energy, Part C 5, 409 (1963); D. E. Pritchard, "Cooling neutral atoms in a magnetic trap for precision spectroscopy," Phys. Rev. Lett. 51, 1336 (1983).

5. H. Müntinga et al., "Interferometry with Bose-Einstein condensates in microgravity," Phys. Rev. Lett. 110, 093602 (2013). 
6. P A. Hogan and S. O'Farrell, "Modelling background radiation in isotropic cosmologies," Gen. Relativ. Gravit. 43, 1625

(2011).

7. G. Rosi et al., "Precision measurement of the Newtonian gravitational constant using cold atoms," Nature 510, 518 (2014).

8. P. Asenbaum et al., "Phase shift in an atom interferometer due to spacetime curvature across its wave function," Phys. Rev. Lett. 118, 183602 (2017).

9. P. W. Graham et al., "Vector dark matter from inflationary fluctuations," Phys. Rev. D 93, 103520 (2016).

10. T. Schuldt et al., "Design of a dual species atom interferometer for space," Exper. Astron. 39, 167 (2015). 